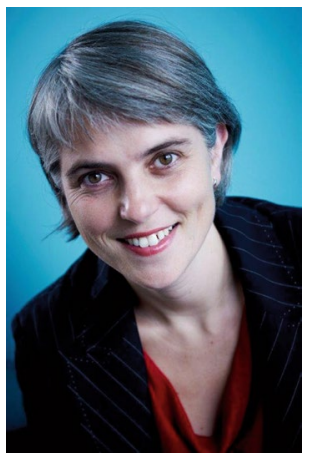

\title{
The United Nations Decade of Healthy Ageing requires concerted global action
}

\author{
Actions are needed by national and local governments, businesses and community \\ organizations to rise to the challenge of the age shift as part of the UN Decade of \\ Healthy Ageing.
}

T he world is undergoing a huge age shift. According to the United Nations Population profiles, the number of people aged 60 and over is projected to increase in high-income countries from 302 million to more than 366 million people between 2019 and 2030, while the number of children aged 0-14 years old will decline from 211 to 202 million. Most of us can expect to live many years longer than our parents' and grandparents' generations. These longer lives are a huge achievement driven by improvements in public health and health care. But many societies across the world are not prepared for the implications of the age shift and have not taken the action needed to ensure that people are able to age well. In the UK, for example, while life expectancy continues to rise, gains in disability-free life expectancy have stalled, meaning people are spending more years in poor health or disability.

The Decade of Healthy Ageing (2020-2030) is a unique and vital opportunity to bring together all sections of society to improve the lives of older people today and future generations. In 2015, the World Health Organization's landmark report on aging and health (https://bit.ly/3ln $86 \mathrm{Ag}$ ) set out a framework for healthy aging that made it clear that health and wellbeing in later life is not just about maintaining physical and mental health, but is also about creating an environment that enables us to live our lives to the full. Five years on, the Decade of Healthy Ageing aims to promote action globally to combat ageism, create more age friendly cities and communities, and secure integrated health care and long-term care so more people can live a healthy and active later life.

At the Centre for Ageing Better (https:// www.ageing-better.org.uk/), we create change in policy and practice informed by evidence and work with partners across England to ensure everyone is able to enjoy later life. 'Healthy aging' is one of our four priority areas, and our goal is for people to have five more years of preventable disability and to reduce the gap between the richest and poorest people in disability-free life expectancy by 2035 . This means working to prevent the onset of poor health - for example, by tackling physical inactivity - but it also means tackling the wider determinants of health such as work, housing and the built environment.

Success will require national governments to adopt and implement strategies on aging that recognize that this is not simply an issue of pensions and social care, but needs action from across government. It will also need local leadership to ensure that the places where people live enable them to stay active and participate as they age. Finally, it will require cross-sector collaboration, with businesses and the voluntary sector working together to develop innovative solutions that have economic and social benefits.

The UK has shown leadership on this issue in recent years. In 2018, $£ 300$ million was announced for a landmark aging society grand challenge (https://bit. ly/2VhpCvh), as part of the UK's Industrial Strategy (https://bit.ly/3o6sQhG), to develop innovative solutions to meet the challenges of an aging population. In the same year, the government committed to our goal of improving disability-free life expectancy and reducing inequalities (https://bit.ly/37jshdI). Unfortunately, as a result of COVID-19, some momentum has been lost in this area and it's vital that we use the opportunity of the Decade to galvanize continued action. The UK should follow the example of other countries such as Singapore, Australia and New Zealand, all of which have national aging strategies that aim to promote healthy and productive aging.

The UK Network of Age-friendly Communities (https://bit.ly/33uv02L) is an example of local leadership and collaboration in action. Launched in 2012 and supported by the Centre for Ageing Better, the network brings together places that are committed to becoming age-friendly communities. Working at a local level with citizens, network members are developing new initiatives - from the Isle of Wight's age-friendly training for bus drivers to Leeds's 'shared tables' initiative for older people living alone. Through the network they share good practice and learn from one another. The UK network is currently made up of over 40 cities, towns, districts and counties, and is linked with the World Health Organization's global network of over 1,000 age-friendly cities and communities. This kind of local leadership and global collaboration will be vital to accelerate progress over the coming decade by enabling places to exchange ideas and practical solutions.

The coronavirus pandemic has had a devastating impact on people's lives, both on their health and their finances, and highlighted huge inequalities in how well we age and therefore how resilient we are to these shocks. The pandemic will continue to present serious challenges for countries across the world for many years to come. But the crisis has also forced governments, businesses and society to rapidly change and adapt in some positive ways, too. Greater flexibility in working patterns could enable more older workers to remain in employment for longer; local mutual aid groups, formed within days, were able to offer fast and targeted support to those who needed it. Now we have a vital opportunity to do things differently. As we enter the Decade of Healthy Ageing, we need governments, businesses and community organizations across the world to rise to the challenge of the age shift and to take action so that everyone is able to live healthier and happier longer lives.

\section{Anna Dixon (iD) \\ Centre for Ageing Better, London, UK.

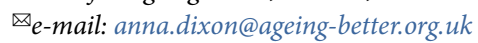

Published online: 14 January 2021 https://doi.org/10.1038/s43587-020-00011-5

Competing interests

The author declares no competing interests. 